\title{
LIMIT OF THE HAUSDORFF DISTANCE FOR ONE-PARAMETER FAMILIES OF WULFF SHAPES CONSTRUCTED BY AFFINE PERTURBATIONS OF DUAL WULFF SHAPES*
}

\author{
HUHE $\mathrm{HAN}^{\dagger}$ AND TAKASHI NISHIMURA ${ }^{\ddagger}$
}

Abstract. It is known that the Wulff construction is an isometry. In this paper we provide an alternative proof of this fact. Moreover, according to this result we investigate the limit of the Hausdorff distance for one-parameter families of Wulff shapes constructed by affine perturbations of dual Wulff shapes.

Key words. Wulff shape, Pompeiu-Hausdorff metric, Maximum distance, dual Wulff shape.

Mathematics Subject Classification. 47N10, 52A30, 82D25.

1. Introduction. Throughout this paper, we let $n, S^{n}$ and $\mathbb{R}_{+}$be a positive integer, the unit sphere of $\mathbb{R}^{n+1}$ and the set consisting of positive real numbers respectively. Define the set $C^{0}\left(S^{n}, \mathbb{R}_{+}\right)$as follows

$$
C^{0}\left(S^{n}, \mathbb{R}_{+}\right)=\left\{\gamma: S^{n} \rightarrow \mathbb{R}_{+} \mid \gamma \text { is continuous }\right\} .
$$

For any $\gamma \in C^{0}\left(S^{n}, \mathbb{R}_{+}\right)$and any $\theta \in S^{n}$, let $\Gamma_{\gamma, \theta}$ be the following half-space, where the dot in the center stands for the scalar product of two vectors $x, \theta \in \mathbb{R}^{n+1}$.

$$
\Gamma_{\gamma, \theta}=\left\{x \in \mathbb{R}^{n+1} \mid x \cdot \theta \leq \gamma(\theta)\right\}
$$

The Wulff shape associated with $\gamma$, denoted by $\mathcal{W}_{\gamma}$, is the following intersection

$$
\mathcal{W}_{\gamma}=\bigcap_{\theta \in S^{n}} \Gamma_{\gamma, \theta} .
$$

This construction is well-known as the Wulff construction of geometric model for an equilibrium crystal introduced by G. Wulff in [9]. By definition, it is clear that Wulff shape is a convex body containing the origin of $\mathbb{R}^{n+1}$ as an interior point. Conversely, it is known that any convex body containing the origin as an interior point is a Wulff shape associated with an appropriate continuous function ([8]). For details on Wulff shapes, see for example $[2,5,6,8]$.

Given a $\gamma \in C^{0}\left(S^{n}, \mathbb{R}_{+}\right)$, set

$$
\operatorname{graph}(\gamma)=\left\{(\theta, \gamma(\theta)) \in \mathbb{R}^{n+1}-\{\mathbf{0}\} \mid \theta \in S^{n}\right\}
$$

where $(\theta, \gamma(\theta))$ is the polar plot expression for a point of $\mathbb{R}^{n+1}-\{\mathbf{0}\}$. The mapping inv : $\mathbb{R}^{n+1}-\{\mathbf{0}\} \rightarrow \mathbb{R}^{n+1}-\{\mathbf{0}\}$, defined as follows, is called the inversion with respect to the origin of $\mathbb{R}^{n+1}$.

$$
\operatorname{inv}(\theta, r)=\left(-\theta, \frac{1}{r}\right)
$$

\footnotetext{
*Received May 31, 2018; accepted for publication August 6, 2018.

${ }^{\dagger}$ College of Science, Northwest Agriculture and Forestry University, China (han-huhe@nwafu. edu.cn).

${ }^{\ddagger}$ Research Group of Mathematical Sciences, Research Institute of Environment and Information Sciences, Yokohama National University, Yokohama 240-8501, Japan (nishimura-takashiyx@ynu.ac.jp). The second author was partially supported by JSPS KAKENHI Grant Number JP17K05245.
} 
Let $\Gamma_{\gamma}$ be the boundary of the convex hull of $\operatorname{inv}(\operatorname{graph}(\gamma))$. If the equality $\Gamma_{\gamma}=$ $\operatorname{inv}(\operatorname{graph}(\gamma))$ is satisfied, then $\gamma$ is called a convex integrand. If the convex hull of $\operatorname{inv}(\operatorname{graph}(\gamma))$ is a strictly convex body and the equality $\Gamma_{\gamma}=\operatorname{inv}(\operatorname{graph}(\gamma))$ is satisfied, then $\gamma$ is called a strictly convex integrand. We called the convex hull of $\operatorname{inv}(\operatorname{graph}(\gamma))$ is the dual Wulff shape of $\mathcal{W}_{\gamma}$. The notion of convex integrand was firstly introduced by J. Taylor in [8] and it plays a key role for studying Wulff shapes (for details on convex integrands, see for instance $[5,8]$ ). Let $C I\left(S^{n}, \mathbb{R}_{+}\right)$be the set consisting of convex integrands.

$$
C I\left(S^{n}, \mathbb{R}_{+}\right)=\left\{\gamma \in C^{0}\left(S^{n}, \mathbb{R}_{+}\right) \mid \gamma: \text { convex integrand }\right\} .
$$

Let $\mathcal{H}_{\text {conv }, \mathbf{0}}\left(\mathbb{R}^{n+1}\right)$ be the set consisting of convex bodies containing the origin of $\mathbb{R}^{n+1}$ as an interior point.

$$
\begin{aligned}
& \mathcal{H}_{\text {conv }, \mathbf{0}}\left(\mathbb{R}^{n+1}\right) \\
= & \left\{W \subset \mathbb{R}^{n+1} \mid W: \text { convex body and } \mathbf{0} \in \mathbb{R}^{n+1} \text { is an intrior point of } \mathcal{W}\right\} .
\end{aligned}
$$

Then, the mapping $\mathcal{W}: C^{0}\left(S^{n}, \mathbb{R}_{+}\right) \rightarrow \mathcal{H}_{\text {conv }, \mathbf{0}}\left(\mathbb{R}^{n+1}\right)$, defined by

$$
\mathcal{W}(\gamma)=\mathcal{W}_{\gamma}
$$

is well-defined. The space $C^{0}\left(S^{n}, \mathbb{R}_{+}\right)$(resp., $\left.\mathcal{H}_{\text {conv }, \mathbf{0}}\left(\mathbb{R}^{n+1}\right)\right)$ is a metric space with respect to the maximum distance (resp., the Pompeiu-Hausdorff distance). For details on the Pompeiu-Hausdorff distance see for instance [1]. It is not difficult to see that the restriction of $\mathcal{W}$ to $C I\left(S^{n}, \mathbb{R}_{+}\right)$is continuous and bijective. Let

$$
C^{0}\left(C I\left(S^{n}, \mathbb{R}_{+}\right), \mathcal{H}_{\text {conv }, \mathbf{0}}\left(\mathbb{R}^{n+1}\right)\right)
$$

be the set consisting of continuous mappings from $C I\left(S^{n}, \mathbb{R}_{+}\right)$into $\mathcal{H}_{\text {conv, } \mathbf{0}}\left(\mathbb{R}^{n+1}\right)$ with respect to the maximum distance and the Pompeiu-Hausdorff distance respectively.

In [7], it is shown that the restriction of $\mathcal{W}$ to $C I\left(S^{n}, \mathbb{R}_{+}\right)$is an isometry. In this paper we first give an alternative proof of this result (see Section 3).

TheOREM $1([7])$. The restriction of $\mathcal{W}$ to $C I\left(S^{n}, \mathbb{R}_{+}\right)$,

$$
\left.\mathcal{W}\right|_{C I\left(S^{n}, \mathbb{R}_{+}\right)}: C I\left(S^{n}, \mathbb{R}_{+}\right) \rightarrow \mathcal{H}_{\text {conv }, \mathbf{0}}\left(\mathbb{R}^{n+1}\right),
$$

is an isometry.

According to this result, we investigate the limit of the Hausdorff distance for oneparameter families of Wulff shapes constructed by affine perturbations of dual Wulff shapes. There are several steps for our investigation. Firstly, we construct a strictly convex integrand as follows. For any $\mathbf{a} \in \mathbb{R}^{n+1}-\{\mathbf{0}\}$, any positive real number $c$ such that $c>\|\mathbf{a}\|$, we consider the function $f_{\mathbf{a}, c}: S^{n} \rightarrow \mathbb{R}$ defined by

$$
f_{\mathbf{a}, c}(X)=\mathbf{a} \cdot X+c .
$$

Then, as shown in p. 237 of [4], $f_{\mathbf{a}, c}$ we have the following:

\section{Proposition 1.}

(1) For each $i, f_{\mathbf{a}_{i}, c_{i}}$ is stable and $\operatorname{Sing}\left(f_{\mathbf{a}_{i}, c_{i}}\right)$, which consists of Morse singular points, is the set $\left\{\frac{\mathbf{a}_{i}}{\left\|\mathbf{a}_{i}\right\|},-\frac{\mathbf{a}_{i}}{\left\|\mathbf{a}_{i}\right\|}\right\}$. 
(2) Suppose that $\mathbf{a}_{1}, \mathbf{a}_{2}$ are linearly independent. Then, the mapping $\left(f_{\mathbf{a}_{1}, c_{1}}, f_{\mathbf{a}_{2}, c_{2}}\right): S^{n} \rightarrow \mathbb{R}^{2}$ is stable and Sing $\left(\left(f_{\mathbf{a}_{1}, c_{1}}, f_{\mathbf{a}_{2}, c_{2}}\right)\right)$, which consists of definite fold points, is just the circle $S^{n} \cap\left(\mathbb{R} \mathbf{a}_{1}+\mathbb{R} \mathbf{a}_{2}\right)$.

(3) Suppose that $\mathbf{a}_{1}, \mathbf{a}_{2}$ are linearly dependent. Then, the mapping $\left(f_{\mathbf{a}_{1}, c_{1}}, f_{\mathbf{a}_{2}, c_{2}}\right)$ : $S^{n} \rightarrow \mathbb{R}^{2}$ may be regarded as a stable function, and $\operatorname{Sing}\left(\left(f_{\mathbf{a}_{1}, c_{1}}, f_{\mathbf{a}_{2}, c_{2}}\right)\right)$, which consists of Morse singular points, is the $\operatorname{set}\left\{\frac{\mathbf{a}_{1}}{\left\|\mathbf{a}_{1}\right\|},-\frac{\mathbf{a}_{1}}{\left\|\mathbf{a}_{1}\right\|}\right\}$.

We concentrate on $f_{\mathbf{a}, c}$ from the viewpoint of convexity. We have the inquality $\mathbf{a} \cdot X+c=\|a\| \cos \theta+c>0$, where $\theta$ is the angle between vectors a and $X$ in $\mathbb{R}^{n+1}$. Thus, $f_{\mathbf{a}, c}: S^{n} \rightarrow \mathbb{R}$ is a positive function (for details, see the proof of Proposition $2)$.

Proposition 2. For any $i \in\{1,2\}$, suppose that $\frac{3}{2}\left\|\mathbf{a}_{i}\right\|<c_{i}$. Let $g_{\mathbf{a}_{i}, c_{i}}: S^{n} \rightarrow$ $\mathbb{R}_{+}$be defined by $g_{\mathbf{a}_{i}, c_{i}}(X)=\sqrt{f_{\mathbf{a}_{i}, c_{i}}(X)}$. Then, $\operatorname{graph}\left(g_{\mathbf{a}_{i}, c_{i}}\right)$ is the boundary of a strictly convex body.

Corollary 1. For any $i$, suppose that $\frac{3}{2}\left\|\mathbf{a}_{i}\right\|<c_{i}$. Define $\frac{1}{g_{\mathbf{a}_{i}}, c_{i}}: S^{n} \rightarrow \mathbb{R}_{+}$by $\frac{1}{g_{\mathbf{a}_{i}}, c_{i}}(X)=\frac{1}{\sqrt{f_{\mathbf{a}_{i}, c_{i}}(X)}}$. Then, $\frac{1}{g_{\mathbf{a}_{i}}, c_{i}}$ is a strictly convex integrand.

Nextly, for any two given convex integrands $\gamma_{1}, \gamma_{2}$ and any $\mathbf{a}_{\mathbf{1}}, \mathbf{a}_{\mathbf{2}} \in \mathbb{R}^{n+1}-\{\mathbf{0}\}$, $c_{1}>\frac{3}{2}\left\|\mathbf{a}_{1}\right\|, c_{2}>\frac{3}{2}\left\|\mathbf{a}_{\mathbf{2}}\right\|$, we consider the following functions $\Gamma_{1, t}, \Gamma_{2, t}: S^{n} \rightarrow \mathbb{R}_{+}$ defined as follows,

$$
\Gamma_{1, t}(X)=\frac{t \gamma_{1}}{1+t \gamma_{1} g_{\mathbf{a}_{1}, c_{1}}}, \quad \Gamma_{2, t}(X)=\frac{t \gamma_{2}}{1+t \gamma_{2} g_{\mathbf{a}_{2}, c_{2}}},
$$

where $t \in(0, \infty)$. Since $g_{\mathbf{a}_{i}, c_{i}}(i=1,2)$ is a strictly convex integrand by Corollary 1 , it is not difficult to show that the function $\Gamma_{1, t}$ and $\Gamma_{2, t}$ are strictly convex integrand, for any sufficiently large $t \in(0, \infty)$ (see Proposition 5 in Section 2).

PRoposition 3. Let $\gamma_{1}, \gamma_{2}$ be convex integrands. Let $g_{\mathbf{a}_{1}, c_{1}}, g_{\mathbf{a}_{2}, c_{2}}$ be as above for $\mathbf{a}_{\mathbf{1}}, \mathbf{a}_{\mathbf{2}} \in \mathbb{R}^{n+1}-\{\mathbf{0}\}$ and $c_{1}>\frac{3}{2}\left\|\mathbf{a}_{\mathbf{1}}\right\|, c_{2}>\frac{3}{2}\left\|\mathbf{a}_{\mathbf{2}}\right\|$. Then we have the following.

(1) $\lim _{t \rightarrow \infty} \mathcal{W}_{\Gamma_{i, t}}=\mathcal{W}_{\lim _{t \rightarrow \infty} \Gamma_{i, t}}=\mathcal{W}_{\frac{1}{g_{\mathbf{a}}, c_{i}}} \quad(i=1,2)$,

(2) $\lim _{t \rightarrow \infty} h\left(\mathcal{W}_{\Gamma_{1, t}}, \mathcal{W}_{\Gamma_{2, t}}\right)=d\left(\frac{1}{g_{\mathbf{a}_{1}, c_{1}}}, \frac{1}{g_{\mathbf{a}_{2}, c_{2}}}\right)$.

By Proposition 3, it follows that the Hausdorff distance of the limit of oneparameter families of the Wulff shapes $\left\{\mathcal{W}_{\Gamma_{i, t}}\right\}(t \rightarrow \infty)$ does not depend on the given $\gamma_{i}$, it does depend only on the given convex integrand $g_{\mathbf{a}_{i}, c_{i}}$. Moreover, we have the following result.

TheOREM 2. Let $\mathbf{a}_{1}, \mathbf{a}_{2}$ be two vectors of $\mathbb{R}^{n+1}-\{\mathbf{0}\}$ and let $c_{1}, c_{2}$ be positive real numbers such that $\frac{3}{2}\left\|\mathbf{a}_{i}\right\|<c_{i}$ for each $i$. Let $g_{\mathbf{a}_{i}, c_{i}}: S^{n} \rightarrow \mathbb{R}_{+}$be the function defined by $g_{\mathbf{a}_{i}, c_{i}}(X)=\sqrt{\mathbf{a}_{i} \cdot X+c_{i}}$. Then, the following equality holds:

$$
d\left(\frac{1}{g_{\mathbf{a}_{1}, c_{1}}}, \frac{1}{g_{\mathbf{a}_{2}, c_{2}}}\right)=\max \left\{\left|\frac{1}{\sqrt{\mathbf{a}_{1} \cdot X+c_{1}}}-\frac{1}{\sqrt{\mathbf{a}_{2} \cdot X+c_{2}}}\right| \mid X \in S^{n} \cap\left(\mathbb{R} \mathbf{a}_{1}+\mathbb{R} \mathbf{a}_{2}\right)\right\} .
$$

This paper is organized as follows. In Section 2, preliminaries are given. The proofs of Proposition 2 and Proposition 3 are given in Section 2. The proof of Theorem 1 (resp., Theorem 2) is given in Section 3 (resp., Section 4). In the Section 5, an alternative proof of Theorem 2 and some examples are given. 


\section{Preliminaries.}

Proof of Proposition 2. We would like to show that $\operatorname{graph}\left(g_{\mathbf{a}_{i}, c_{i}}\right)$ is the boundary of a strictly convex body. In order to do so, take two distinct point $P, Q \in S^{n}$ so that $P \neq-Q$. Let $\xi: I=(p, q) \rightarrow S^{n}$ be the geodesic with arc-length parameter such that $\lim _{s \rightarrow p} \xi(s)=P$ and $\lim _{s \rightarrow q} \xi(s)=Q$. Then, it is sufficient to show that the curvature of $g_{\mathbf{a}_{i}, c_{i}} \circ \xi(s)$ is always positive for any $s \in I$. Since $\xi$ is a geodesic of $S^{n}$, its image $\xi(I)$ is contained in a plane. Hence, from the first we may assume that $\xi$ is a map $I \rightarrow S^{1}$. Thus, it is sufficient to show that for the function $g_{i}: I \rightarrow \mathbb{R}$ with the form $g_{i}(\theta)=\sqrt{\lambda_{i} \cos \theta+\mu_{i} \sin \theta+c_{i}}$ where $\lambda_{i}=\mathbf{a}_{i} \cdot P, \mu_{i}=\mathbf{a}_{i} \cdot \frac{Q-(P \cdot Q) P}{\|Q-(P \cdot Q) P\|}$ and for any $\theta \in I$,

$$
\frac{g_{i}^{2}(\theta)+2{g_{i}^{\prime}}^{2}(\theta)-g_{i}(\theta) g_{i}^{\prime \prime}(\theta)}{\left(g_{i}^{2}(\theta)+g_{i}^{\prime 2}(\theta)\right)^{\frac{3}{2}}}>0 .
$$

The numerator of the above formula is as follows.

$$
\begin{aligned}
& g_{i}^{2}(\theta)+2 g_{i}^{\prime 2}(\theta)-g_{i}(\theta) g_{i}^{\prime \prime}(\theta) \\
= & \left(\lambda_{i} \cos \theta+\mu_{i} \sin \theta+c_{i}\right)+2\left(\frac{-\lambda_{i} \sin \theta+\mu_{i} \cos \theta}{2 g_{i}(\theta)}\right)^{2} \\
& \quad-g_{i}(\theta) \frac{2\left(-\lambda_{i} \cos \theta-\mu_{i} \sin \theta\right) g_{i}^{2}(\theta)-\left(-\lambda_{i} \sin \theta+\mu_{i} \cos \theta\right)^{2}}{4 g_{i}^{3}(\theta)} \\
= & \frac{3}{2}\left(\lambda_{i} \cos \theta+\mu_{i} \sin \theta\right)+c_{i}+\frac{3\left(-\lambda_{i} \sin \theta+\mu_{i} \cos \theta\right)^{2}}{4 g_{i}^{2}(\theta)} \\
\geq & \frac{3}{2}\left(\lambda_{i} \cos \theta+\mu_{i} \sin \theta\right)+c_{i} \\
\geq & -\frac{3}{2}\left\|\mathbf{a}_{i}\right\|+c_{i}>0 .
\end{aligned}
$$

Therefore, $(*)$ is always positive.

Since a convex integrand $\gamma$ is of class $C^{1}$ if and only if its Wulff shape is a strictly convex body ([3]), by Proposition 2, we have the following.

Proposition 4. Let $\gamma: S^{n} \rightarrow \mathbb{R}_{+}$be a convex integrand, a $\in \mathbb{R}^{n+1}-\{\mathbf{0}\}$ and $c>\frac{3}{2}\|\mathbf{a}\|$. Then the boundary of the Wulff shape $\mathcal{W}_{\frac{\gamma}{1+\gamma g_{\mathbf{a}, c}}}$ is of class $C^{1}$.

Next, for given convex integrands $\gamma_{1}, \gamma_{2}$ and $\mathbf{a}_{\mathbf{1}}, \mathbf{a}_{\mathbf{2}} \in \mathbb{R}^{n+1}-\{\mathbf{0}\}, c_{1}>$ $\frac{3}{2}\left\|\mathbf{a}_{\mathbf{1}}\right\|, c_{2}>\frac{3}{2}\left\|\mathbf{a}_{\mathbf{2}}\right\|$, we consider the convex integrands $\Gamma_{1, t}, \Gamma_{2, t}: S^{n} \rightarrow \mathbb{R}_{+}$defined as follows, where $t \in(0, \infty)$.

$$
\Gamma_{1, t}(X)=\frac{t \gamma_{1}(X)}{1+t \gamma_{1}(X) g_{\mathbf{a}_{1}, c_{1}}(X)}, \Gamma_{2, t}(X)=\frac{t \gamma_{2}(X)}{1+t \gamma_{2}(X) g_{\mathbf{a}_{2}, c_{2}}(X)}
$$

Proposition 5. Let $t \in \mathbb{R}_{+}$be sufficiently large. Then, for any $i \in\{1,2\}, \Gamma_{i, t}$ is always a strictly convex integrand.

Proof. Remember that

$$
\frac{1}{\Gamma_{i, t}(X)}=\frac{1}{t}\left(\frac{1}{\gamma_{i}(X)}+\operatorname{tg}_{\mathbf{a}_{i}, c_{i}}(X)\right) .
$$


Similarly as in the proof of Proposition 2, we would like to show that if $t$ is sufficiently large, then

$$
\operatorname{graph}\left(\frac{1}{\gamma_{i}}+t g_{\mathbf{a}_{i}, c_{i}}\right)
$$

is the boundary of a strictly convex body. In order to do so, it is sufficient to show that there exists a positive number $T$ such that for any $t$ satisfying $t>T$ and any two distinct points $P, Q \in S^{n}$ such that $P \neq-Q$ and the geodesic $\xi: I \rightarrow S^{n}$ with arc-length parameter connecting $P$ and $Q$, the curvature of

$$
\frac{1}{\gamma_{i} \circ \xi}+\operatorname{tg}_{\mathbf{a}_{i}, c_{i}} \circ \xi
$$

is always positive. Again similarly as in the proof of Proposition 2, from the first we may assume that $\xi$ is a mapping $I \rightarrow S^{1}$. Moreover, for simplicity, we set

$$
f=\frac{1}{\gamma_{i} \circ \xi(s)}, g=g_{\mathbf{a}_{i}, c_{i}} \circ \xi(s) .
$$

Then, it is sufficient to show that there exists a positive number $T$ such that for any $t$ satisfying $t>T$ and any two distinct points $P, Q \in S^{n}$ such that $P \neq-Q$, the geodesic $\xi: I \rightarrow S^{n}$ with arc-length parameter connecting $P, Q$ and any $s \in I$,

$$
\frac{(f+t g)^{2}+2\left(f^{\prime}+t g^{\prime}\right)^{2}-(f+t g)\left(f^{\prime \prime}+t g^{\prime \prime}\right)}{\left((f+t g)^{2}+\left(f^{\prime}+t g^{\prime}\right)^{2}\right)^{\frac{3}{2}}}>0 .
$$

The denominator of $(* *)$ is always positive. We concentrate on its numerator. Since $f^{2}+2 f^{\prime 2}-f f^{\prime \prime}$ is always non-negative and $g^{2}+2 g^{\prime 2}-g g$ " is always positive, we have the following:

$$
\begin{aligned}
& (f+t g)^{2}+2\left(f^{\prime}+t g^{\prime}\right)^{2}-(f+t g)\left(f^{\prime \prime}+t g^{\prime \prime}\right) \\
= & \left(g^{2}+2 g^{\prime 2}-g g^{\prime \prime}\right) t^{2}+2\left(f g+2 f^{\prime} g^{\prime}-f^{\prime \prime} g-f g^{\prime \prime}\right) t \\
& +\left(f^{2}+2 f^{\prime 2}-f f^{\prime \prime}\right) \\
\geq & t\left(g^{2}+2 g^{\prime 2}-g g^{\prime \prime}\right)\left(t+2 \frac{f g+2 f^{\prime} g^{\prime}-f^{\prime \prime} g-f g^{\prime \prime}}{g^{2}+2 g^{\prime 2}-g g^{\prime \prime}}\right) .
\end{aligned}
$$

Let $C$ be a great circle of $S^{n}$. For the $C$, set

$$
M_{C}=\min _{X \in C} 2 \frac{f g+2 f^{\prime} g^{\prime}-f^{\prime \prime} g-f g^{\prime \prime}}{g^{2}+2 g^{2}-g g^{\prime \prime}} .
$$

Of course, the value $M_{C}$ depends on $C$. In other words, the function

$$
M: V_{2}\left(\mathbb{R}^{n+1}\right) \rightarrow \mathbb{R}
$$

defined by $M\left(\left(\mathbf{v}_{1}, \mathbf{v}_{2}\right)\right)=M_{C}$ is a well-defined continuous function, where $V_{2}\left(\mathbb{R}^{n+1}\right)$ is the Stiefel manifold consisting of orthonormal 2-frames in $\mathbb{R}^{n+1}$ and $C=$ $\left(\mathbb{R} \mathbf{v}_{1}+\mathbb{R} \mathbf{v}_{2}\right) \cap S^{n}$. Since $V_{2}\left(\mathbb{R}^{n+1}\right)$ is compact, the image of $M$, too, is a compact set. Denote its minimal value by $m$.

Set $T=|m|$. Then, for any $t$ satisfying $t>T$, any two distinct points $P, Q \in S^{n}$ such that $P \neq-Q$, the geodesic $\xi: I \rightarrow S^{n}$ connecting $P, Q$ and any $s \in I$,

$$
\frac{(f+t g)^{2}+2\left(f^{\prime}+t g^{\prime}\right)^{2}-(f+t g)\left(f^{\prime \prime}+t g^{\prime \prime}\right)}{\left((f+t g)^{2}+\left(f^{\prime}+t g^{\prime}\right)^{2}\right)^{\frac{3}{2}}}>0 .
$$


Proof of Proposition 3. Since the space consisting of non-empty compact set of $\mathbb{R}^{n+1}$ is a complete metric space with respect to the Pompeiu-Hausdorff distance and the mapping $\mathcal{W}$ is continuous, the following equality holds.

$$
\lim _{t \rightarrow \infty} \mathcal{W}_{\Gamma_{i, t}}=\mathcal{W}_{\lim _{i} \rightarrow \infty \Gamma_{i, t}} . \quad(i=1,2)
$$

Also, since

$$
\lim _{t \rightarrow \infty} \Gamma_{i, t}=\lim _{t \rightarrow \infty} \frac{1}{\frac{1}{t \gamma_{i}}+g_{\mathbf{a}_{\mathbf{i}}, c_{i}}}=\frac{1}{g_{\mathbf{a}_{\mathrm{i}}, c_{i}}}
$$

the assertion (1)

$$
\lim _{t \rightarrow \infty} \mathcal{W}_{\Gamma_{i, t}}=\mathcal{W}_{\lim _{t \rightarrow \infty} \Gamma_{i, t}}=\mathcal{W}_{\overline{g_{\mathbf{i}}, c_{i}}} \quad(i=1,2)
$$

holds.

By Theorem 1, the assertion (2) easily follows from the following equality.

$$
\lim _{t \rightarrow \infty} h\left(\mathcal{W}_{\Gamma_{1, t}}, \mathcal{W}_{\Gamma_{2, t}}\right)=\lim _{t \rightarrow \infty} d\left(\frac{t \gamma_{1}}{1+t \gamma_{1} g_{\mathbf{a}_{1}, c_{1}}}, \frac{t \gamma_{2}}{1+t \gamma_{2} g_{\mathbf{a}_{2}, c_{2}}}\right)=d\left(\frac{1}{g_{\mathbf{a}_{1}, c_{1}}}, \frac{1}{g_{\mathbf{a}_{2}, c_{2}}}\right) .
$$

\section{Proof of Theorem 1.}

LEMMA 3.1. Given a convex integrand $\gamma \in C I\left(S^{n}, \mathbb{R}_{+}\right)$and a positive real number $a \in \mathbb{R}_{+}$, define the continuous function $\gamma_{a}: S^{n} \rightarrow \mathbb{R}_{+}$by $\gamma_{a}(\theta)=\gamma(\theta)+$ a for any $\theta \in S^{n}$. Then, the following equality holds.

$$
\mathcal{W}_{\gamma, a}=\overline{B\left(\mathcal{W}_{\gamma}, a\right)}
$$

Here, $\overline{B\left(\mathcal{W}_{\gamma}, a\right)}$ means $\bigcup_{P \in \mathcal{W}_{\gamma}} \overline{B(P, a)}=\bigcup_{P \in \mathcal{W}_{\gamma}}\left\{x \in \mathbb{R}^{n+1} \mid\|x-P\| \leq a\right\}$.

Proof. We first prove the inclusion $\overline{B\left(\mathcal{W}_{\gamma}, a\right)} \subset \mathcal{W}_{\gamma_{a}}$. Suppose that there exists a point $P$ of the boundary of $\overline{B\left(\mathcal{W}_{\gamma}, a\right)}$ such that $P$ is not included in $\mathcal{W}_{\gamma_{a}}$. Then, by the definition of Wulff shape, there exists a point $\theta$ of $S^{n}$ such that the following sharp inequality holds,

$$
\gamma(\theta)+a<P \cdot \theta
$$

Let $Q$ be a point of the boundary of $\mathcal{W}_{\gamma}$ such that $d(P, Q)=a$. It is clear that there exists a point $Q$ such that $d(P, Q) \leq a$. Suppose that there exists a point $Q$ of the boundary of $\mathcal{W}_{\gamma}$ such that $d(P, Q)<a$. Then, there exists a positive real number $\varepsilon$ satisfying $\overline{B(P, \varepsilon)} \subset \overline{B(Q, a)}$. This means $\overline{B(P, \varepsilon)} \subset \overline{B\left(\mathcal{W}_{\gamma}, a\right)}$, which contradicts the fact that $P$ is a point of the boundary of $\overline{B\left(\mathcal{W}_{\gamma}, a\right)}$. Thus, by the sharp inequality $\gamma(\theta)+a<P \cdot \theta$, the following holds.

$$
\gamma(\theta)+a<P \cdot \theta=\left(Q+a \frac{P-Q}{\|P-Q\|}\right) \cdot \theta=Q \cdot \theta+\left(a \frac{P-Q}{\|P-Q\|}\right) \cdot \theta .
$$

On the other hand, it is clear that the following holds for any $\theta$ of $S^{n}$.

$$
Q \cdot \theta \leq \gamma(\theta) \text { and }\left(a \frac{P-Q}{\|P-Q\|}\right) \cdot \theta \leq a .
$$




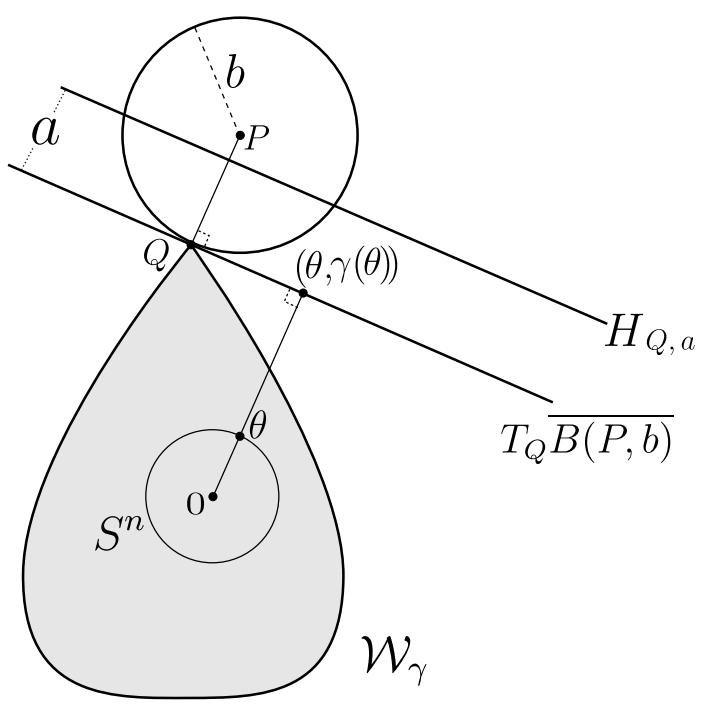

FIG. 1. The hyperplanes $T_{Q} \overline{B(P, b)}$ and $H_{Q, a}$.

Thus, we have the following.

$$
Q \cdot \theta+\left(a \frac{P-Q}{\|P-Q\|}\right) \cdot \theta \leq \gamma(\theta)+a .
$$

This contradicts $(* * *)$.

Next, we prove the converse inclusion $\mathcal{W}_{\gamma_{a}} \subset \overline{B\left(\mathcal{W}_{\gamma}, a\right)}$. Suppose that there exists a point $P$ of $\mathcal{W}_{\gamma_{a}}$ such that $P$ is not included in $\overline{B\left(\mathcal{W}_{\gamma}, a\right)}$. Then the intersection of $\overline{B(P, a)}$ and $\mathcal{W}_{\gamma}$ is the empty set. Let $b$ be the positive real number such that the following equality holds.

$$
\overline{B(P, b)} \cap \partial \mathcal{W}_{\gamma}=\{Q\}
$$

It is clear that $a<b=\|P-Q\|$. Let $T_{Q} \overline{B(P, b)}$ be the affine tangent hyperplane to $\overline{B(P, b)}$ at $Q$ (see Figure 1 ). Since $\mathcal{W}_{\gamma}$ is a convex body, it follows that $T_{Q} \overline{B(P, b)}$ is a support hyperplane to $\mathcal{W}_{\gamma}$ at $Q$. This meas that $\mathcal{W}_{\gamma} \cap T_{Q} \overline{B(P, b)}$ is a subset of the boundary of $\mathcal{W}_{\gamma}$. Set

$$
\theta=\frac{P-Q}{\|P-Q\|}
$$

Then $\theta$ is a point of $S^{n}$. Notice that there exists a positive real number $\lambda$ such that $P-Q=\lambda \theta$. It follows that $Q \cdot \theta=\gamma(\theta)$ and $(\theta, \gamma(\theta)) \in T_{Q} \overline{B(P, b)}$. By the assumption, the hyperplane

$$
H_{Q, a}=\left\{R \in \mathbb{R}^{n+1} \mid R=M+a \frac{P-Q}{\|P-Q\|}, M \in T_{Q} \overline{B(P, b)}\right\}
$$

does not contain the point $Q$ and the intersection of the segment $P Q$ and $H_{Q, a}$ is not empty (see Figure 1). Thus the following sharp inequality holds,

$$
\gamma(\theta)+a=Q \cdot \theta+\left(a \frac{P-Q}{\|P-Q\|}\right) \cdot \theta<Q \cdot \theta+\left(b \frac{P-Q}{\|P-Q\|}\right) \cdot \theta=P \cdot \theta
$$


Since $P$ is a point of $\mathcal{W}_{\gamma_{a}},(* *)$ contradicts the inequality $P \cdot \theta \leq \gamma_{a}(\theta)$.

Now we are ready to prove Theorem 1 . It is enough to show the following two.

(1) $h\left(\mathcal{W}_{\gamma_{1}}, \mathcal{W}_{\gamma_{2}}\right) \leq d\left(\gamma_{1}, \gamma_{2}\right)$ for any $\gamma_{1}, \gamma_{2} \in C I\left(S^{n}, \mathbb{R}_{+}\right)$.

(2) $d\left(\gamma_{1}, \gamma_{2}\right) \leq h\left(\mathcal{W}_{\gamma_{1}}, \mathcal{W}_{\gamma_{2}}\right)$ for any $\gamma_{1}, \gamma_{2} \in C I\left(S^{n}, \mathbb{R}_{+}\right)$.

Suppose that (1) does not hold. Then, there exist two convex integrands $\gamma_{1}, \gamma_{2}$ such that the sharp inequality $d\left(\gamma_{1}, \gamma_{2}\right)<h\left(\mathcal{W}_{\gamma_{1}}, \mathcal{W}_{\gamma_{2}}\right)$ holds. Set $d\left(\gamma_{1}, \gamma_{2}\right)=a>0$. By Lemma 3.1, there exists a point $P$ of $\mathcal{W}_{\gamma_{1}}$ such that

$$
P \notin \overline{B\left(\mathcal{W}_{\gamma_{2}}, a\right)}=\mathcal{W}_{\gamma_{2, a}} .
$$

Then by the definition of Wulff shape, there exists a point $\theta$ of $S^{n}$ such that the inequality $\gamma_{2}(\theta)+a=\gamma_{2, a}(\theta)<P \cdot \theta$ holds. On the other hand, since $P$ is a point of $\mathcal{W}_{\gamma_{1}}$, we have that $P \cdot \theta \leq \gamma_{1}(\theta)$ for any $\theta$ of $S^{n}$. By the assumption, it follows that

$$
\gamma_{2}(\theta)+a<P \cdot \theta \leq \gamma_{1}(\theta)<\gamma_{2}(\theta)+a
$$

Thus, we have a contradiction.

Next, we show the inequality (2). Suppose that there exist two convex integrands $\gamma_{1}, \gamma_{2}$ such that the sharp inequality $h\left(\mathcal{W}_{\gamma_{1}}, \mathcal{W}_{\gamma_{2}}\right)<d\left(\gamma_{1}, \gamma_{2}\right)$ holds. Set $h\left(\mathcal{W}_{\gamma_{1}}, \mathcal{W}_{\gamma_{2}}\right)=a>0$. By the definition of maximum distance, there exists a point $\theta$ of $S^{n}$ satisfying $a<\left|\gamma_{1}(\theta)-\gamma_{2}(\theta)\right|$. Then, without loss of generality, we may assume that $a<\gamma_{1}(\theta)-\gamma_{2}(\theta)$. Notice that for any $\theta$ of $S^{n}$, there exists a point $P_{\theta}$ of the boundary of $\mathcal{W}$ such that the equality $P_{\theta} \cdot \theta=\gamma_{\mathcal{W}}(\theta)$ holds. It follows that, for the $\theta$ of $S^{n}$ satisfying $a<\gamma_{1}(\theta)-\gamma_{2}(\theta)$, there exists a point $P_{\theta}$ of the boundary of $\mathcal{W}_{\gamma_{1}}$ such that the following holds,

$$
\gamma_{2}(\theta)+a<\gamma_{1}(\theta)=P_{\theta} \cdot \theta
$$

By Lemma 3.1, it follows that

$$
P_{\theta} \notin \mathcal{W}_{\gamma_{2, a}}=\overline{B\left(\mathcal{W}_{\gamma_{2}}, a\right)}
$$

This contradicts the assumption $h\left(\mathcal{W}_{\gamma_{1}}, \mathcal{W}_{\gamma_{2}}\right)=a$. Therefore, the restriction of $\mathcal{W}$ to $C I\left(S^{n}, \mathbb{R}_{+}\right)$is an isometry.

By Theorem 1, we clearly have the following (see Figure 2).

Corollary 2. Let $\mathcal{W}_{1}, \mathcal{W}_{2}$ be two elements of $\mathcal{H}_{\mathrm{conv}, \mathbf{0}}\left(\mathbb{R}^{n+1}\right)$ and let $T: \mathbb{R}^{n+1} \rightarrow$ $\mathbb{R}^{n+1}$ be a parallel translation such that the origin $\mathbf{0}$ is an interior point of $T\left(\mathcal{W}_{1}\right) \cap$ $T\left(\mathcal{W}_{2}\right)$. Then, the following equality holds.

$$
d\left(\gamma_{\mathcal{W}_{1}}, \gamma_{\mathcal{W}_{2}}\right)=d\left(\gamma_{T\left(\mathcal{W}_{1}\right)}, \gamma_{T\left(\mathcal{W}_{2}\right)}\right)
$$

For any $c \in \mathbb{R}_{+}$, let $c^{*}: S^{n} \rightarrow \mathbb{R}_{+}$be the constant function $c^{*}\left(S^{n}\right)=c$. For any $\mathcal{W} \in \mathcal{H}_{\text {conv }, \mathbf{0}}\left(\mathbb{R}^{n+1}\right)$, define the function $w^{*}: S^{n} \rightarrow \mathbb{R}_{+}$by $w^{*}(\theta)=\min \{c \in$ $\left.\mathbb{R}_{+} \mid \mathcal{W} \subset \Gamma_{c^{*}, \theta}\right\}$ for any $\theta \in S^{n}$. Then, notice that $\gamma_{\mathcal{W}}=w^{*}$. By this observation, the following is clearly obtained as a corollary of Theorem 1 .

Corollary 3. Let $W_{1}, W_{2}$ be two convex bodies such that the intersection $W_{1} \cap$ $W_{2}$ is a convex body. Then, $h\left(W_{1}, W_{2}\right)$ can be calculated radially from any interior point of $W_{1} \cap W_{2}$. More precisely, the following holds.

$$
\begin{aligned}
& h\left(W_{1}, W_{2}\right) \\
= & \max _{\theta \in S^{n}}\left|\min \left\{c \in \mathbb{R}_{+} \mid W_{1} \subset\left(x+\Gamma_{c^{*}, \theta}\right)\right\}-\min \left\{c \in \mathbb{R}_{+} \mid W_{2} \subset\left(x+\Gamma_{c^{*}, \theta}\right)\right\}\right|,
\end{aligned}
$$



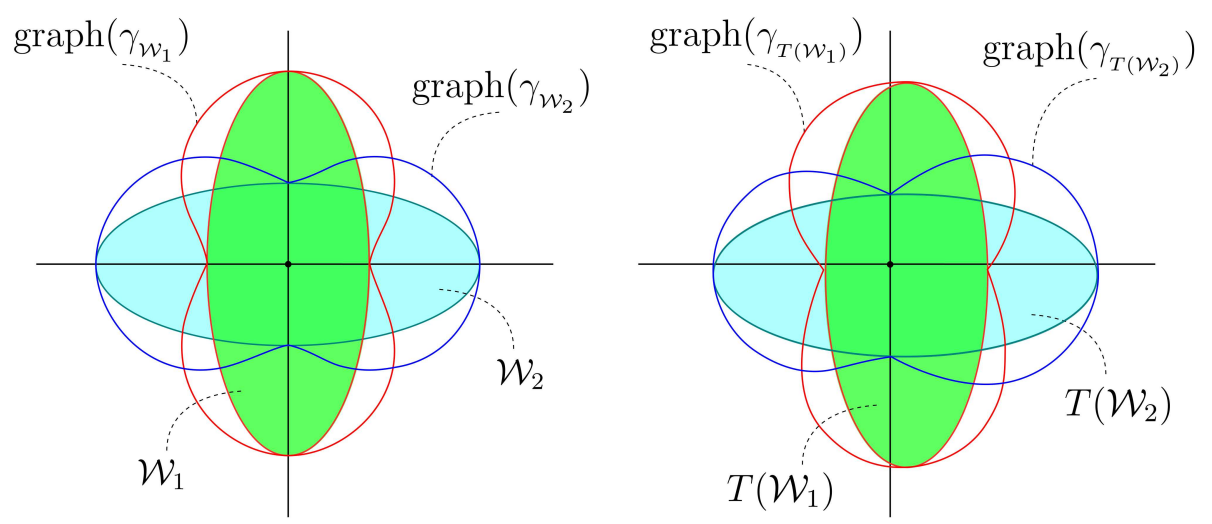

FIG. 2. $d\left(\gamma_{\mathcal{W}_{1}}, \gamma_{\mathcal{W}_{2}}\right)=d\left(\gamma_{T\left(\mathcal{W}_{1}\right)}, \gamma_{T\left(\mathcal{W}_{2}\right)}\right)$.

where $x$ is an interior point of $W_{1} \cap W_{2}$.

For given two convex bodies such that their intersection is a convex body, Corollary 3 gives an algorithm that can compute an approximate value of the Pompeiu-Hausdorff distance with high precision.

\section{Proof of Theorem 2. Since}

$$
\lim _{t \rightarrow \infty} \Gamma_{i, t}=\lim _{t \rightarrow \infty} \frac{t \gamma_{i}}{1+t \gamma_{i} g_{\mathbf{a}_{i}, c_{i}}}=\lim _{t \rightarrow \infty} \frac{\gamma_{i}}{\frac{1}{t}+\gamma_{i} g_{\mathbf{a}_{i}, c_{i}}}=\frac{1}{g_{\mathbf{a}_{i}, c_{i}}},
$$

by Corollary 1 (the Wulff construction is continuous) and Proposition $2\left(\frac{1}{g_{\mathbf{a}_{i}, c_{i}}}\right.$ is a strictly convex integrand if $\left.\frac{3}{2}\left\|\mathbf{a}_{i}\right\|<c_{i}\right)$, the following holds:

$$
\lim _{t \rightarrow \infty} \mathcal{W}\left(\Gamma_{i, t}\right)=\mathcal{W}\left(\lim _{t \rightarrow \infty} \Gamma_{i, t}\right)=\mathcal{W} \frac{1}{g_{\mathbf{a}_{\mathbf{i}}, c_{i}}} \quad(i=1,2)
$$

Hence we have

$$
\begin{aligned}
\lim _{t \rightarrow \infty} h\left(\mathcal{W}\left(\Gamma_{1, t}\right), \mathcal{W}\left(\Gamma_{2, t}\right)\right) & =h\left(\lim _{t \rightarrow \infty} \mathcal{W}\left(\Gamma_{1, t}\right), \lim _{t \rightarrow \infty} \mathcal{W}\left(\Gamma_{2, t}\right)\right) \\
& =h\left(\mathcal{W}_{\overline{g_{\mathbf{a}_{1}, c_{1}}}}, \mathcal{W}_{\overline{g_{\mathbf{a}_{2}}, c_{2}}}\right) .
\end{aligned}
$$

Therefore, by Theorem $1\left(h\left(\mathcal{W}_{\gamma_{1}}, \mathcal{W}_{\gamma_{2}}\right)=d\left(\gamma_{1}, \gamma_{2}\right)\right.$ for convex integrands $\left.\gamma_{1}, \gamma_{2}\right)$,

$$
\lim _{t \rightarrow \infty} h\left(\mathcal{W}\left(\Gamma_{1, t}\right), \mathcal{W}\left(\Gamma_{2, t}\right)\right)=d\left(\frac{1}{g_{\mathbf{a}_{1}, c_{1}}}, \frac{1}{g_{\mathbf{a}_{2}, c_{2}}}\right) .
$$

Next, we consider the mapping $\left(g_{\mathbf{a}_{1}, c_{1}}, g_{\mathbf{a}_{2}, c_{2}}\right): S^{n} \rightarrow \mathbb{R}^{2}$. Set $G=\left(g_{\mathbf{a}_{1}, c_{1}}, g_{\mathbf{a}_{2}, c_{2}}\right)$, $F=\left(f_{\mathbf{a}_{1}, c_{1}}, f_{\mathbf{a}_{2}, c_{2}}\right)$. Let $H: \mathbb{R}_{+}^{2} \rightarrow \mathbb{R}_{+}^{2}$ be the $C^{\infty}$ diffeomorphism defined by $H\left(X_{1}, X_{2}\right)=\left(\sqrt{X_{1}}, \sqrt{X_{2}}\right)$. Then, it is clear that

$$
G=H \circ F \text {. }
$$

Hence, $H$ gives the correspondence between the boundary of $G\left(S^{n}\right)$ and the boundary of $F\left(S^{n}\right)$. On the other hand, by definition, we have the following:

$$
d\left(\frac{1}{g_{\mathbf{a}_{1}, c_{1}}}, \frac{1}{g_{\mathbf{a}_{2}, c_{2}}}\right) \in\left\{\left|\frac{1}{X_{1}}-\frac{1}{X_{2}}\right| \mid\left(X_{1}, X_{2}\right) \in \partial G\left(S^{n}\right)\right\},
$$


where $\partial G\left(S^{n}\right)$ stands for the boundary of $G\left(S^{n}\right)$. Moreover, by Proposition 1 (Any projection of $S^{n}$ is stable), $\partial F\left(S^{n}\right)$ is characterized as follows:

$$
\partial F\left(S^{n}\right)=\left\{F(\theta) \mid \theta \in S^{n} \cap\left(\mathbb{R} \mathbf{a}_{1}+\mathbb{R} \mathbf{a}_{2}\right)\right\} .
$$

Therefore, as a conclusion, we have the following:

$$
\begin{aligned}
& \lim _{t \rightarrow \infty} h\left(\mathcal{W}_{\Gamma_{1, t}}, \mathcal{W}_{\Gamma_{2, t}}\right) \\
= & d\left(\frac{1}{g_{\mathbf{a}_{1}, c_{1}}}, \frac{1}{g_{\mathbf{a}_{2}, c_{2}}}\right) \\
= & \max \left\{\left|\frac{1}{g_{\mathbf{a}_{1}, c_{1}}(\theta)}-\frac{1}{g_{\mathbf{a}_{2}, c_{2}}(\theta)}\right| \mid \theta \in S^{n} \cap\left(\mathbb{R} \mathbf{a}_{1}+\mathbb{R} \mathbf{a}_{2}\right)\right\} .
\end{aligned}
$$

5. Appendix. Since the function $\frac{1}{g_{\mathbf{a}, c}}: S^{n} \rightarrow \mathbb{R}_{+}$defined by

$$
\frac{1}{g_{\mathbf{a}, c}}(X)=\frac{1}{\sqrt{f_{\mathbf{a}, c}(X)}},
$$

$\mathbf{a} \in \mathbb{R}^{n+1}-\{\mathbf{0}\}$ and $c>\frac{3}{2}\|\mathbf{a}\|$, we have that

$$
d\left(\frac{1}{g_{\mathbf{a}, c_{1}}}, \frac{1}{g_{\mathbf{b}, c_{2}}}\right)=\max \left\{\left|\left(\mathbf{a} \cdot X+c_{1}\right)^{-\frac{1}{2}}-\left(\mathbf{b} \cdot X+c_{2}\right)^{-\frac{1}{2}}\right| X \in S^{n}\right\} .
$$

Let $g: S^{n} \rightarrow \mathbb{R}$ be a mapping defined by $g(X)=X \cdot X-1$. Let $F: S^{n} \rightarrow \mathbb{R}$ be a mapping defined by

$$
F(X)=\left(\mathbf{a} \cdot X+c_{1}\right)^{-\frac{1}{2}}-\left(\mathbf{b} \cdot X+c_{2}\right)^{-\frac{1}{2}}-\lambda g(X),
$$

where $\lambda$ is a constant. Set $\mathbf{a}=\left\{a_{1}, \ldots, a_{n+1}\right\}, \mathbf{b}=\left\{b_{1}, \ldots, b_{n+1}\right\}$. If $X$ is a local conditional extrema point under the constraints $g(X)=0$, by method of Lagrange multipliers, the following equalities holds.

$$
\left\{\begin{array}{c}
F_{x_{1}}=-\frac{1}{2}\left(\mathbf{a} \cdot X+c_{1}\right)^{-\frac{3}{2}} a_{1}+\frac{1}{2}\left(\mathbf{b} \cdot X+c_{2}\right)^{-\frac{3}{2}} b_{1}-2 \lambda x_{1}=0 \\
F_{x_{2}}=-\frac{1}{2}\left(\mathbf{a} \cdot X+c_{1}\right)^{-\frac{3}{2}} a_{2}+\frac{1}{2}\left(\mathbf{b} \cdot X+c_{2}\right)^{-\frac{3}{2}} b_{2}-2 \lambda x_{2}=0 \\
\vdots \\
\vdots \\
F_{x_{n+1}}=-\frac{1}{2}\left(\mathbf{a} \cdot X+c_{1}\right)^{-\frac{3}{2}} a_{n+1}+\frac{1}{2}\left(\mathbf{b} \cdot X+c_{2}\right)^{-\frac{3}{2}} b_{n+1}-2 \lambda x_{n+1}=0 \\
F_{\lambda}=x_{1}^{2}+x_{2}^{2}+\cdots+x_{n+1}^{2}-1=0 .
\end{array}\right.
$$

The equalities $(\checkmark)$ can also be written as follows.

$$
\left\{\begin{array}{l}
-\frac{1}{2}\left(\mathbf{a} \cdot X+c_{1}\right)^{-\frac{3}{2}} \mathbf{a}+\frac{1}{2}\left(\mathbf{b} \cdot X+c_{2}\right)^{-\frac{3}{2}} \mathbf{b}=2 \lambda X \\
x_{1}^{2}+x_{2}^{2}+\cdots+x_{n+1}^{2}-1=0
\end{array}\right.
$$

This implies that if $X$ is a local conditional extrema point under the constraint $g(X)=$ 0 then the vectors $\mathbf{a}, \mathbf{b}$ and $X$ are linearly dependent. So we have that

$$
\begin{aligned}
d\left(\frac{1}{g_{\mathbf{a}, c_{1}}}, \frac{1}{g_{\mathbf{b}, c_{2}}}\right) & =\max \left\{\mid\left(\mathbf{a} \cdot X+c_{1}\right)^{-\frac{1}{2}}-\left(\mathbf{b} \cdot X+c_{2}\right)^{-\frac{1}{2}} \| X \in S^{n}\right\} \\
& =\max \left\{\mid\left(\mathbf{a} \cdot X+c_{1}\right)^{-\frac{1}{2}}-\left(\mathbf{b} \cdot X+c_{2}\right)^{-\frac{1}{2}} \| X \in S^{n} \cap(\mathbb{R} \mathbf{a}+\mathbb{R} \mathbf{b})\right\} .
\end{aligned}
$$


5.1. Some special case of Theorem 2. In the case of $\mathbf{a}=\mathbf{b}$. In this case, we can write the equalities

$$
\left\{\begin{array}{l}
F_{x_{1}}=-\frac{1}{2}\left(\mathbf{a} \cdot X+c_{1}\right)^{-\frac{3}{2}} a_{1}+\frac{1}{2}\left(\mathbf{a} \cdot X+c_{2}\right)^{-\frac{3}{2}} a_{1}-2 \lambda x_{1}=0 \\
F_{x_{2}}=-\frac{1}{2}\left(\mathbf{a} \cdot X+c_{1}\right)^{-\frac{3}{2}} a_{2}+\frac{1}{2}\left(\mathbf{a} \cdot X+c_{2}\right)^{-\frac{3}{2}} a_{2}-2 \lambda x_{2}=0 \\
\quad \vdots \quad \vdots \\
F_{x_{n+1}}=-\frac{1}{2}\left(\mathbf{a} \cdot X+c_{1}\right)^{-\frac{3}{2}} a_{n+1}+\frac{1}{2}\left(\mathbf{a} \cdot X+c_{2}\right)^{-\frac{3}{2}} a_{n+1}-2 \lambda x_{n+1}=0, \\
F_{\lambda}=x_{1}^{2}+x_{2}^{2}+\cdots+x_{n+1}^{2}-1=0 .
\end{array}\right.
$$

Since $-\frac{1}{2}\left(\mathbf{a} \cdot X+c_{1}\right)^{-\frac{3}{2}}+\frac{1}{2}\left(\mathbf{a} \cdot X+c_{2}\right)^{-\frac{3}{2}}$ is never be zero, it follows that

$$
\left\{\begin{array}{l}
\frac{a_{1}}{x_{1}}=\frac{a_{2}}{x_{2}}=\cdots=\frac{a_{n+1}}{x_{n+1}}, \\
x_{1}^{2}+x_{2}^{2}+\cdots+x_{n+1}^{2}-1=0 .
\end{array}\right.
$$

This implies that if $X$ is a local conditional extrema point under the constraint $g(X)=$ 0 , then vectors $X$ and $\mathbf{a}$ are linearly dependent. So in this case $\mathbf{a}=\mathbf{b}$, we have that

$$
\begin{gathered}
d\left(\frac{1}{g_{\mathbf{a}, c_{1}}}, \frac{1}{g_{\mathbf{b}, c_{2}}}\right)=\max \left\{\left|\left(\mathbf{a} \cdot X+c_{1}\right)^{-\frac{1}{2}}-\left(\mathbf{b} \cdot X+c_{2}\right)^{-\frac{1}{2}}\right| \mid X \in S^{n}\right\} \\
=\max \left\{\left|\left(|| \mathbf{a} \|+c_{1}\right)^{-\frac{1}{2}}-\left(\|\mathbf{a}\|+c_{2}\right)^{-\frac{1}{2}}\right|,\right. \\
\left.\left|\left(-\|\mathbf{a}\|+c_{1}\right)^{-\frac{1}{2}}-\left(-\|\mathbf{a}\|+c_{2}\right)^{-\frac{1}{2}}\right|\right\} .
\end{gathered}
$$

ExAmple. Let $n=1, \mathbf{a}_{1}=\mathbf{a}_{2} \in \mathbb{R}^{2}-\{\mathbf{0}\}$ and $\frac{3}{2}\left\|\mathbf{a}_{\mathbf{1}}\right\|<c_{2}<c_{1}$. Let $X=$ $(\cos \theta, \sin \theta) \in S^{1}$, We consider the mapping $G: S^{n} \rightarrow \mathbb{R}^{2}-\{\mathbf{0}\}$ defined as

$$
G(X)=\left(\frac{1}{g_{\mathbf{a}_{1}, c_{1}}(X)}, \frac{1}{g_{\mathbf{a}_{2}, c_{2}}(X)}\right) .
$$

Let $K$ is the positive real number such that $d\left(\frac{1}{g_{\mathbf{a}_{1}, c_{1}}}, \frac{1}{g_{\mathbf{a}_{2}, c_{2}}}\right)=K$. Then for any $X \in S^{1}$, we have that $d\left(\frac{1}{g_{\mathbf{a}_{1}, c_{1}}(X)}, \frac{1}{g_{\mathbf{a}_{2}, c_{2}}(X)}\right) \leq K$. This implies that

the lines $y=x-K$ or $y=x+K$ is tangent to image of $G$ at the

point $\left(\frac{1}{g_{\mathbf{a}_{1}, c_{1}}(X)}, \frac{1}{g_{\mathbf{a}_{2}, c_{2}}(X)}\right)$ such that $d\left(\frac{1}{g_{\mathbf{a}_{1}, c_{1}}}, \frac{1}{g_{\mathbf{a}_{2}, c_{2}}}\right)=\left|\frac{1}{g_{\mathbf{a}_{1}, c_{1}}(X)}-\frac{1}{g_{\mathbf{a}_{2}, c_{2}(X)}}\right|$.

Moreover, by assumption, we know that the point $X \in S^{1}$ gives the distance $d\left(\frac{1}{g_{\mathbf{a}_{1}, c_{1}}}, \frac{1}{g_{\mathbf{a}_{2}, c_{2}}}\right)$ satisfies the equality

$$
\left(\frac{1}{g_{\mathbf{a}_{1}, c_{1}}(X)}\right)^{\prime}=\left(\frac{1}{g_{\mathbf{a}_{2}, c_{2}}(X)}\right)^{\prime}
$$

Since

$$
\begin{aligned}
\frac{d}{d \theta} G(\cos \theta, \sin \theta)=( & -\frac{1}{2}\left(a_{1} \cos \theta+a_{2} \sin \theta+c_{1}\right)^{-\frac{3}{2}}\left(-a_{1} \sin \theta+a_{2} \cos \theta\right), \\
& \left.-\frac{1}{2}\left(a_{1} \cos \theta+a_{2} \sin \theta+c_{2}\right)^{-\frac{3}{2}}\left(-a_{1} \sin \theta+a_{2} \cos \theta\right)\right),
\end{aligned}
$$


by the equality $(\star \star)$, we know that the point gives the distance $d\left(\frac{1}{g_{\mathbf{a}, c_{1}}}, \frac{1}{g_{\mathbf{a}, c_{2}}}\right)$ is satisfies the following equality,

$$
\begin{aligned}
& -\frac{1}{2}\left(a_{1} \cos \theta+a_{2} \sin \theta+c_{1}\right)^{-\frac{3}{2}}\left(-a_{1} \sin \theta+a_{2} \cos \theta\right) \\
= & -\frac{1}{2}\left(a_{1} \cos \theta+a_{2} \sin \theta+c_{2}\right)^{-\frac{3}{2}}\left(-a_{1} \sin \theta+a_{2} \cos \theta\right) .
\end{aligned}
$$

This implies

$$
\begin{array}{r}
-\frac{1}{2}\left(\left(a_{1} \cos \theta+a_{2} \sin \theta+c_{1}\right)^{-\frac{3}{2}}-\left(a_{1} \cos \theta+a_{2} \sin \theta+c_{2}\right)^{-\frac{3}{2}}\right) \\
\left(-a_{1} \sin \theta+a_{2} \cos \theta\right)=0 .
\end{array}
$$

Since the part $-\frac{1}{2}\left(\left(a_{1} \cos \theta+a_{2} \sin \theta+c_{1}\right)^{-\frac{3}{2}}-\left(a_{1} \cos \theta+a_{2} \sin \theta+c_{2}\right)^{-\frac{3}{2}}\right)$ is always non zero real number for any $(\cos \theta, \sin \theta) \in S^{1}$, the equality $(*)$ holds if and only if $\left(-a_{1} \sin \theta+a_{2} \cos \theta\right)=0$, namely, the singular point of mapping $G$. It follows that the point $(\cos \theta, \sin \theta)$ gives $d\left(\frac{1}{g_{\mathbf{a}_{1}, c_{1}}}, \frac{1}{g_{\mathbf{a}_{\mathbf{2}}, c_{2}}}\right)$ then there exists a real number $m$ such that $(\cos \theta, \sin \theta)=m\left(a_{1}, a_{2}\right)$.

In the case of $k \mathbf{a}=\mathbf{b}, k$ is a real number. In this case, we can write the equalities $(\checkmark)$ as

$$
\left\{\begin{array}{c}
F_{x_{1}}=-\frac{1}{2}\left(\mathbf{a} \cdot X+c_{1}\right)^{-\frac{3}{2}} a_{1}+\frac{1}{2}\left(k \mathbf{a} \cdot X+c_{2}\right)^{-\frac{3}{2}} k a_{1}-2 \lambda x_{1}=0 \\
F_{x_{2}}=-\frac{1}{2}\left(\mathbf{a} \cdot X+c_{1}\right)^{-\frac{3}{2}} a_{2}+\frac{1}{2}\left(k \mathbf{a} \cdot X+c_{2}\right)^{-\frac{3}{2}} k a_{2}-2 \lambda x_{2}=0 \\
\vdots \quad \vdots \\
F_{x_{n+1}}=-\frac{1}{2}\left(\mathbf{a} \cdot X+c_{1}\right)^{-\frac{3}{2}} a_{n+1}+\frac{1}{2}\left(k \mathbf{a} \cdot X+c_{2}\right)^{-\frac{3}{2}} k a_{n+1}-2 \lambda x_{n+1}=0 \\
F_{\lambda}=x_{1}^{2}+x_{2}^{2}+\cdots+x_{n+1}^{2}-1=0
\end{array}\right.
$$

In the same way, we have that

$$
\begin{aligned}
& d\left(\frac{1}{g_{\mathbf{a}, c_{1}}}, \frac{1}{g_{\mathbf{b}, c_{2}}}\right)= \max \left\{\left|\left(\mathbf{a} \cdot X+c_{1}\right)^{-\frac{1}{2}}-\left(\mathbf{b} \cdot X+c_{2}\right)^{-\frac{1}{2}}\right| X \in S^{n}\right\} \\
&=\max \left\{\left|\left(\|\mathbf{a}\|+c_{1}\right)^{-\frac{1}{2}}-\left(k\|\mathbf{a}\|+c_{2}\right)^{-\frac{1}{2}}\right|,\right. \\
&\left.\left|\left(-\|\mathbf{a}\|+c_{1}\right)^{-\frac{1}{2}}-\left(-k\|\mathbf{a}\|+c_{2}\right)^{-\frac{1}{2}}\right|\right\} .
\end{aligned}
$$

In the case of $\mathbf{a}=(m, 0, \ldots, 0)$ and $\mathbf{b}=(0, \ldots, 0, r)$, where $m, r$ are non-zero real numbers. In this case, we can write the equalities $(\bullet)$ as

$$
\left\{\begin{array}{l}
F_{x_{1}}=-\frac{m}{2}\left(x_{1}+c_{1}\right)^{-\frac{3}{2}}-2 \lambda x_{1}=0 \\
F_{x_{2}}=-2 \lambda x_{2}=0 \\
\quad \vdots \\
F_{x_{n+1}}=-\frac{r}{2}\left(x_{n+1}+c_{2}\right)^{-\frac{3}{2}}-2 \lambda x_{n+1}=0 \\
F_{\lambda}=x_{1}^{2}+x_{2}^{2}+\cdots+x_{n+1}^{2}-1=0
\end{array}\right.
$$


This implies that if $X$ is a local conditional extrema point under the constraints $g(X)=0$, it follows that

$$
\left\{\begin{array}{l}
-\frac{m}{2}\left(x_{1}+c_{1}\right)^{-\frac{3}{2}}-2 \lambda x_{1}=0 \\
x_{2}=\cdots=x_{n}=0 \\
-\frac{r}{2}\left(x_{n+1}+c_{2}\right)^{-\frac{3}{2}}-2 \lambda x_{n+1}=0 \\
x_{1}^{2}+x_{n+1}^{2}-1=0
\end{array}\right.
$$

Therefore the vectors $\mathbf{a}, \mathbf{b}$ and $X$ are linearly dependent and $\mathbf{a} \cdot \mathbf{b}=0$. Set $\cos \theta=\mathbf{a} \cdot X$ and $\sin \theta=\mathbf{b} \cdot X$. Then

$$
\begin{aligned}
d\left(\frac{1}{g_{\mathbf{a}, c_{1}}}, \frac{1}{g_{\mathbf{b}, c_{2}}}\right) & =\max \left\{\left|\left(\mathbf{a} \cdot X+c_{1}\right)^{-\frac{1}{2}}-\left(\mathbf{b} \cdot X+c_{2}\right)^{-\frac{1}{2}}\right| X \in S^{n}\right\} \\
& =\max \left\{\left|\left(\cos \theta+c_{1}\right)^{-\frac{1}{2}}-\left(\sin \theta+c_{2}\right)^{-\frac{1}{2}}\right| \theta \in[0, \pi]\right\}
\end{aligned}
$$

Acknowledgement. The authors are very grateful to the referee for careful reading of this paper.

\section{REFERENCES}

[1] M. Barnsley, Fractals Everywhere 2nd edition, Morgan Kaufmann Pub., San Fransisco, 1993.

[2] Y. GigA, Surface Evolution Equations, Monographs of Mathematics, 99, Springer, 2006.

[3] H. Han And T. Nishimura, Strictly convex Wulff shapes and $C^{1}$ convex integrands, Proc. Amer. Math. Soc., 145 (2017), pp. 3997-4008.

[4] J. Mather, Generic projections, Ann. of Math., 98 (1973), pp. 226-245.

[5] F. Morgan, The cone over the Clifford torus in $\mathbb{R}^{4}$ is $\Phi$-minimizing, Math. Ann., 289 (1991), pp. $341-354$.

[6] A. Pimpinelli and J. Villain, Physics of Crystal Growth, Monographs and Texts in Statistical Physics, Cambridge University Press, Cambridge New York, 1998.

[7] R. SchneIder, Convex Bodies: The BrunnMinkowski Theory, Cambridge University Press, 2013

[8] J. E. TAYLOR, Crystalline variational problems, Bull. Amer. Math. Soc., 84 (1978), pp. 568-588.

[9] G. WulfF, Zur frage der geschwindindigkeit des wachstrums und der auflösung der krystallflachen, Z. Kristallographine und Mineralogie, 34 (1901), pp. 449-530. 
H. HAN AND T. NISHIMURA 